\title{
Inlet Conditions for Large Eddy Simulation of Gas-Turbine Swirl Injectors
}

\author{
M. H. Baba-Ahmadi* and G. R. Tabor $\stackrel{\ddagger}{\ddagger}$ \\ University of Exeter, Exeter, England EX4 4QF United Kingdom
}

DOI: $\underline{10.2514 / 1.35259}$

\begin{abstract}
In this paper, we present a novel technique for generating swirl inlets for large eddy simulation. The velocity a short distance downstream of the inlet to the main domain is sampled and the flow velocity data are reintroduced back into the domain inlet, creating an inlet section integrated into the main domain in which turbulence can develop. Additionally, variable artificial body forces and velocity corrections are imposed in this inlet section, with feedback control to force the flow toward desired swirl, mean, and turbulent profiles. The method was applied to flow in an axisymmetric sudden expansion, with and without swirl at the inlet, and compared against experimental and literature large eddy simulation data and against similar results in the literature. The method generates excellent results for this case and is elegant and straightforward to implement.
\end{abstract}

\section{Introduction}

$\mathbf{S}$ WIRL injectors have been widely adopted in combustion systems such as gas-turbine engine combustors to stabilize the flame for efficient and clean combustion. Breakdown of the incoming swirl vortex in the central toroidal recirculation zone creates high shear rates and strong turbulence intensities that act as a flame stabilization mechanism. In addition, the swirl also produces high rates of entrainment and fast mixing. Investigation of these mechanisms is obviously of great interest. Traditionally, designers have relied heavily on empirical correlations for determining overall geometries, dimensions, etc. This approach is now supplemented with theoretical and computational modeling techniques, which have the ability to predict physical phenomena over a wide range of conditions, in addition to providing a better insight into the fluid dynamics. Modeling of these processes, however, is extremely complicated. In particular, swirling flows are difficult to model with Reynolds-averaged Navier-Stokes (RANS) methods due to the effect of the mean flow streamline curvature [1], and so this is one example in which large eddy simulation (LES) methods have come to the fore. However, there are still numerous technical issues to be overcome in implementing LES as a technique [2]. In particular, the provision of adequate boundary conditions (for the case of swirl injection, this particularly means inlet conditions) is one very significant hurdle to be overcome, and this is the subject of the current paper. Implementing inlets for LES is significantly more challenging than is the case for RANS models; the inlet flow has to include the grid-scale (GS) turbulence, and so has to include a stochastically fluctuating component that satisfies a range of conditions (such as the correct temporal and spatial correlation). Thus, the topic of this paper is of great importance for the adoption of LES in this area.

Two approaches to creating inlet conditions for swirling flow have been applied in the literature. The simplest approach is to create a mean flow profile by determining the axial and tangential mean flow components, either from previous computational work (using RANS), from experiment, or from theory, and to impose a specified

Received 22 October 2007; revision received 21 February 2008; accepted for publication 26 February 2008. Copyright () 2008 by the American Institute of Aeronautics and Astronautics, Inc. All rights reserved. Copies of this paper may be made for personal or internal use, on condition that the copier pay the $\$ 10.00$ per-copy fee to the Copyright Clearance Center, Inc., 222 Rosewood Drive, Danvers, MA 01923; include the code 0001-1452/08 $\$ 10.00$ in correspondence with the CCC.

*Senior Lecturer, School of Engineering, Computer Science and Mathematics.

${ }^{\dagger}$ Ph.D. Student, School of Engineering, Computer Science and Mathematics. level of fluctuation on top of this, usually as Gaussian white noise. Examples of this approach include $[3,4]$. However, such approaches suffer problems related to the nonphysical nature of the turbulence introduced at the inlet, leading to incorrect prediction of turbulent kinetic energy and energy spectra downstream of the inlet [5]. Creating an appropriate inlet condition for LES is considerably more challenging than is the case for RANS; because there is no implicit scale separation in LES between simulated and turbulent flow, the grid-scale (explicitly simulated) flow contains a transient component due to turbulent velocity fluctuations, a component that has to be included at the inlet. Moreover, this transient component has to possess most, if not all, of the characteristics of the turbulence that it is representing, including correct spatial and temporal correlation properties, something that white noise fails to satisfy. More sophisticated synthesis techniques have been developed using approaches such as digital filtering and the Fourier series to introduce appropriate correlations [으요, but these have not been applied to swirling flows to date.

The alternative approach to generate a turbulent inlet for LES is via a turbulence-library database. Typically, this involves running a precursor simulation on a simpler geometry (e.g., a cyclic channel), to create fully developed turbulence; successive time steps of this simulation are then saved and replayed into the inlet of the main simulation. Various variants of the technique have been tried, for example, running the precursor simulation in parallel with the main simulation (thus obviating the need to store a limited database of information [9]) and scaling the data using the Reynolds stress (to adjust an existing database to another Reynolds number [10]). In the context of swirling flows, most versions of this technique make use of a method developed by Pierce and Moin [11] for generating swirl within a cyclic channel by imposing a constant tangential body force on the flow. Having computed a library of turbulent swirling flow in this way, either as a saved precursor database or "on the fly" in parallel with the main calculation, the flow conditions from the secondary calculation can be fed into the main computation [12,13]. As an example of this, Wang and Bai [5] used Pierce and Moin's [11] method to create a 10,000 time-step library for lookup, which was then cycled through as appropriate. The library does not, however, meet the specifications for the required flow, and so the data are rescaled to meet the desired statistical properties (specified mean and variance of velocity). However, this rescaling does cause problems; the level of turbulent kinetic energy is seen to decrease downstream of the inlet, which the authors attribute to the unphysical turbulence at the inlet adapting to become true turbulent flow further downstream. Schlüter et al. [14] also implemented and compared various inlet conditions for swirl: specifically, a laminar inflow (no fluctuations), inflow with random fluctuations, and various precomputation methods. As 
before, the laminar and random fluctuation techniques produced poor results, and the various library-lookup techniques performed much better.

In the current work, we present a novel technique for generating an inlet for generating swirl flow for LES by applying a body force and remapping within the main computational domain. Hence, a region of the main computational domain is designated as the inlet region, and at the end of this region, the flow conditions are sampled and fed back into the start of the computational domain. At this level, this approach has been demonstrated to work well in the past $[15,16]$. In addition, axial and tangential body forces are introduced within the inlet section together with velocity-correction terms in this region; feedback control is used to modify these terms to drive the flow toward the desired velocity profiles (including swirl) and turbulence profiles. Our method thus differs from existing published work for swirl inlets in two important details:

1) The turbulence development region is integrated into the main domain rather than having to be run as a separate calculation.

2) The body force is varying rather than fixed and is controlled to very precisely develop the correct mean and turbulence flow conditions.

We also incorporate a correction method to improve the turbulence statistics for the flow, which has been applied before in the literature to generate libraries for lookup [10]; however, this is the first time that this method has been applied directly to the main domain in this manner and applied to generate a swirling flow. Our new approach was applied to the case of incompressible flow over a cylindrical sudden expansion, both with and without swirl at the inlet. This is a canonical test case for swirling flows, in which a jet of fluid with or without swirl enters a much larger cylindrical geometry. In essence, it recreates many of the features of gas-turbine injectors and dump combustors, as well as jets entering larger domains.

\section{Theory}

\section{A. LES and Numerical Implementation}

LES is based on a spatial averaging in the form of a convolution with a spatial filter $G$, separating the flow into GS and subgrid-scale (SGS) components $\mathbf{v}=\overline{\mathbf{v}}+\overline{\mathbf{v}}^{\prime}$, where

$$
\overline{\mathbf{v}}=G \times \mathbf{v}=\int_{D} G(\zeta, \Delta) \mathbf{v}(\zeta, t) \mathrm{d}^{3} \zeta
$$

$\Delta$ is a characteristic scale of $G$, referred to as the filter width, and $D$ is the computational domain. Conventionally, we assume that the filter width is the same as the cell size $\Delta x$, hence the labels grid scale and subgrid scale. In this case, the averaged, or filtered, Navier-Stokes equations take the form

$$
\nabla \cdot \overline{\mathbf{v}}=0, \quad \partial_{t} \overline{\mathbf{v}}+\nabla \cdot(\overline{\mathbf{v}} \otimes \overline{\mathbf{v}})=\nabla \cdot(\overline{\mathbf{S}}-\mathbf{B})+\overline{\mathbf{F}}
$$

given that $[G \times, \nabla] \mathbf{v}=\mathbf{0}: \mathbf{v}$ is the velocity field, $v$ is the molecular viscosity, $\mathbf{S}=-p \mathbf{I}+2 \nu \mathbf{D}$ ( $p$ is the specific pressure), and $\mathbf{D}=\frac{1}{2}\left(\nabla \mathbf{v}+\nabla \mathbf{v}^{T}\right)$. The convolution process generates an additional term, the SGS stress tensor:

$$
\mathbf{B}=\overline{\mathbf{v} \otimes \mathbf{v}}-\overline{\mathbf{v}} \otimes \overline{\mathbf{v}}=\mathbf{L}+\mathbf{C}+\mathbf{R}
$$

where $\mathbf{L}$ is the Leonard stress, $\mathbf{C}$ is the cross stress, and $\mathbf{R}$ is the Reynolds stress tensor [17]. Different modeling of these terms generates the different turbulence models; here, we use the dynamic one-equation model [18]. $\overline{\mathbf{F}}$ represents an artificial body force term that will be discussed next.

The filtered Navier-Stokes equations (1), together with Eq. (2), are solved using the computational fluid dynamics (CFD) OpenFOAM (Field Operation and Manipulation) code library. This is a $\mathrm{C}++$ code library of classes for writing CFD codes, which includes a welltested and validated LES capability [17,19-21]. Equations (1) are discretized using the finite volume method, in which the domain $D$ is divided into cells $\delta V_{i}$ so that

$$
\bigcup_{i}\left(\delta V_{i}\right)=D \cup \partial D
$$

and

$$
\bigcap_{i}\left(\delta V_{i}\right)=\emptyset
$$

Integration of the dependent variables over each cell $\delta V_{i}$, together with application of Gauss's theorem, generates a set of discretized equations with the divergence terms in Eq. (1) represented as fluxes across the cell faces, evaluated using appropriate interpolation schemes; we use centered second-order interpolation and nonvariation-diminishing (NVD) interpolation [gamma scheme (see [22])]. Time integration is carried out by the Crank-Nicholson scheme, which is second order in time. Following the procedure of Rhie and Chow [23], discretization of the $\nabla p$ term is left; a Poisson equation is constructed that implements the incompressibility condition $\nabla \cdot \overline{\mathbf{v}}=0$, and the equation set is solved sequentially using the resulting pressure-implicit splitting of operators (PISO) algorithm [24]. Solution is performed implicitly by matrix inversion using incomplete Cholesky conjugate gradient methods. SGS modeling is provided by the dynamic one-equation model, in which a transport equation is provided for the subgrid turbulent kinetic energy $k$, and the resulting model coefficients can be determined by introducing a second, grid-scale, level of filtering [18]. The oneequation approach may allow for coarser grids than can be used for a comparable problem with a zero-equation model, because some subgrid information is available for the formulation of subgrid-scale models. All of this has been previously implemented in OpenFOAM and extensively validated [17].

\section{B. Inlet Conditions}

Included in the governing equations to be solved is a term $\overline{\mathbf{F}}$, which will be used to drive the flow toward the desired swirl. This term is nonzero within the inlet section of the mesh, in which it takes the value

$$
\overline{\mathbf{F}}=\frac{V_{b}}{L}\left[\alpha\left(\mathbf{v}_{\mathrm{des}}-\langle\overline{\mathbf{v}}\rangle\right)+\left(\mathbf{v}_{\mathrm{des}}-\overline{\mathbf{v}}\right)\right]
$$

where $\mathbf{v}_{\text {des }}$ is the target mean flow profile; $\overline{\mathbf{v}}$ is the instantaneous GS velocity; $\langle\overline{\mathbf{v}}\rangle$ is the time average of the GS velocity, evaluated by taking a running average on the GS velocity; $V_{b}$ and $L$ are the desired bulk velocity and the length of the forcing region, respectively; and $L$ was varied by trial and error to get the best results from the simulation. The value used here of $z / R=4$ corresponds to around 1500 wall units, which should be adequate for turbulence to develop fully.

In Eq. (3), the first term in the bracket [i.e., $\left.\alpha\left(\mathbf{v}_{\text {des }}-\langle\overline{\mathbf{v}}\rangle\right)\right]$ provides feedback control on the mean flow, with $\alpha$ setting the magnitude of the feedback. The second term $\left(\mathbf{v}_{\text {des }}-\overline{\mathbf{v}}\right)$ provides some control over the magnitude of instantaneous fluctuations in the velocity (i.e., the GS turbulence). However, early in the simulation, $\mathbf{v}_{\text {des }}-\langle\overline{\mathbf{v}}\rangle$ is large, with potentially unfortunate consequences. Thus, at the start of the simulation, the value $\alpha=0$ is chosen. As the simulation progresses, $\mathbf{v}_{\text {des }}-\langle\overline{\mathbf{v}}\rangle$ decreases toward zero, and so the value of $\alpha$ is ramped to a maximum value determined so that

$$
\frac{\left|\mathbf{v}_{\mathrm{des}}-\langle\overline{\mathbf{v}}\rangle\right|}{V_{b}}<0.0001
$$

If $\alpha$ were allowed to increase without limit, the force contribution from this term would eventually come to dominate the equation for no physical reason and the simulation would fail.

This control mechanism drives the mean flow toward the desired target $\mathbf{v}_{\text {des }}$ : in this case, the experimentally determined mean flow profiles in the axial and (for the swirling flow) tangential directions. We are also interested in generating the correct turbulence profiles, and to achieve this, a second level of feedback control is provided by correcting the velocity components within the inlet section using the relation 


$$
\overline{\mathbf{v}}^{*}=\mathbf{v}_{\mathrm{des}}+(\overline{\mathbf{v}}-\langle\overline{\mathbf{v}}\rangle) \times\left(\frac{\left(R_{\mathrm{des}}\right)_{i i}}{R_{i i}}\right)^{1 / 2}
$$

where $R_{\text {des }}$ is the desired Reynolds stress and $R$ is the calculated GS Reynolds stress in the mapping section, evaluated by means of a running average. Note that Einstein summation convention is not being used here; we are distinguishing between the Reynolds stress in the three directions, although we are ignoring the cross stresses. The SGS contribution to the calculated Reynolds stress could have been included here, but because we are using an eddy-viscosity SGS model, this would have introduced further assumptions about the isotropy of the turbulence at small length scales. The velocity $\overline{\mathbf{v}}$ is replaced by the updated velocity $\overline{\mathbf{v}}^{*}$ in each cell in the inlet section. Because of the fluctuations, the term $(\overline{\mathbf{v}}-\langle\overline{\mathbf{v}}\rangle)$ is never exactly zero, and so the term $\left(\left(R_{\mathrm{des}}\right)_{i i} / R_{j j}\right)^{1 / 2}$ drives the turbulent fluctuations to provide the target rms mean-velocity components and, in turn, the target axial components of the Reynolds stress. This relation was first implemented in [10] to generate a database for lookup based on an a priori RANS calculation; here, it is applied directly to control the flow. The case being simulated [25] provides experimental measurement of the flow conditions upstream of the inlet, and these were used as the target profiles for the mean and fluctuating velocity components.

\section{Test Case}

The geometry used corresponds to the experiments of Dellenback et al. [25], who undertook measurements in a turbulent swirling flow through an abrupt axisymmetric expansion and examined the influence of the swirl number. Two cases were computed (one without swirl and the other with), both at a Reynolds number of $R e=30,000$, based on the diameter of the nozzle $D$ and the bulk velocity in nozzle $V_{b}$. The level of swirl is expressed in terms of the swirl number, which is the ratio of angular momentum flux in the axial direction to the axial momentum flux in the axial direction; that is,

$$
S=\frac{1}{R} \frac{\int_{0}^{R} r^{2}\left\langle v_{z}\right\rangle\left\langle v_{\theta}\right\rangle \mathrm{d} r}{\int_{0}^{R} r\left\langle v_{z}\right\rangle^{2} \mathrm{~d} r}
$$

where $\left\langle v_{z}\right\rangle$ is the axial velocity component, $\left\langle v_{\theta}\right\rangle$ is the azimuthal velocity component, and $R$ is the radius of the nozzle. The case geometry is shown in Fig. 1 .

The first test was performed for a confined jet without swirl at $R e=30,000$, for which it is well known that the jet spreading rate is strongly dependent on the turbulence present in the jet inflow. The experimental data for this case are available from the Dellenback et al. [25] experiments. The mesh used for this computation consists of a $1 \overline{80} \times 70 \times 70$ cylindrical-block-structured mesh adding up to approximately 850,000 cells, with the smallest cell next to the edge of the jet. The mesh is shown in Fig. $\underline{1}$; as with the pipe meshes, it comprises a core rectangular section surrounded by four blocks that adapt the mesh to the curved inner surface of the geometry. The cell size near the wall upstream of the expansion is approximately $y^{+}=25$, which means that the boundary layer is still under-resolved. The time step was limited by the Courant-Friedrich-Lewy condition
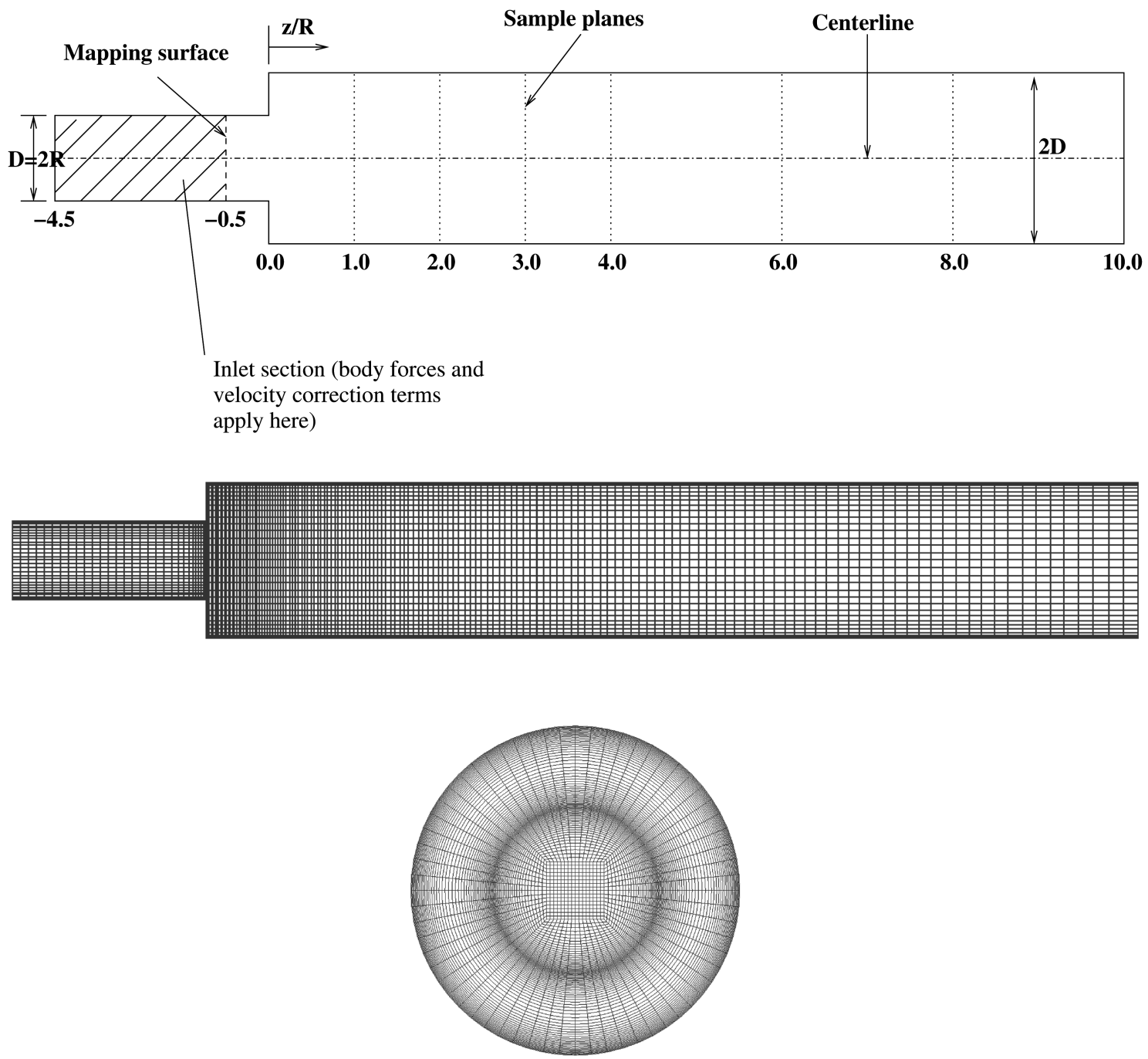

Fig. 1 Geometry used for nonswirling flow in sudden expansion: geometry and location of mapping surface and sample planes (top), cross section through the mesh (middle), and end view of the mesh (bottom). 
(maximum Courant number of 0.5 ) and is approximately $\Delta t=0.025 \mathrm{~s}$. This case was run for $1500 \mathrm{~s}$, which is equivalent to 15 complete transits of the flow through the expansion.

For the second test case, a swirl flow at an expansion with a swirl number $S=0.6$ at $R e=30,000$ was considered. Swirl flows at high swirl numbers $(S>0.25)$ create central recirculation zones [10], and as a result of that, flows with high shear are created that have a high level of turbulence production. The experimental data for this case are also available from the Dellenback et al. [25] experiments. The mesh used for this computation consists of a $248 \times 76 \times 76$ cylindrical-block mesh using approximately 1.5 million cells for computational domain, with the smallest cells next to the edge of the jet. The cell size near the wall upstream of the expansion is approximately $y^{+}=16$, which means that the boundary layer is still under-resolved. The finer mesh required a shorter time step of $\Delta t=0.0125 \mathrm{~s}$. This case was run for $1500 \mathrm{~s}$, equivalent to 30 complete transits of the flow through the domain.

In addition to the experimental data, this test case has been used for LES simulation before; in particular, Schlüter et al. [10] used an LES flow solver developed at the Centre for Turbulence Research [11]. This flow solver solves the filtered momentum equations with a lowMach-number assumption on an axisymmetric structured mesh with a second-order finite volume scheme on a staggered grid. Subgrid stresses are modeled with an eddy-viscosity approach coupled to the dynamic procedure. For inlet conditions, they used a precomputed library modified to account for the unsteadiness of the interface-flow statistics. Numerical results are available for the swirl case and are included in the comparison.

\section{Results}

\section{A. Nonswirl Case}

Figures $\underline{2}-\underline{4}$ show the velocity fields (mean axial velocity $\bar{v}_{z}$ and rms fluctuating components in the axial and tangential directions) obtained for the nonswirl case. Experimental results are shown as dotted lines and the computed results are shown as solid lines. The leftmost velocity profile is located upstream of the expansion and defines the inlet condition for the LES. It can be seen that the simulation reproduces the experimental data well for both mean profiles and turbulent fluctuations. The reattachment of the flow behind the step is also well-predicted, although there is some discrepancy in the tangential rms directly behind the expansion. Figures $\underline{3}$ and $\underline{4}$ show some discrepancies close to the outlet. These can be explained by the fact that LES computations have been shown to be sensitive to outflow conditions $[11,26]$, and so the adjustment of the LES solution near the outlet is very important for these calculations. This matter is particularly important for sudden expansion flows with low-turbulence-inflow conditions. LES outflow conditions have been investigated in detail by Schlüter and

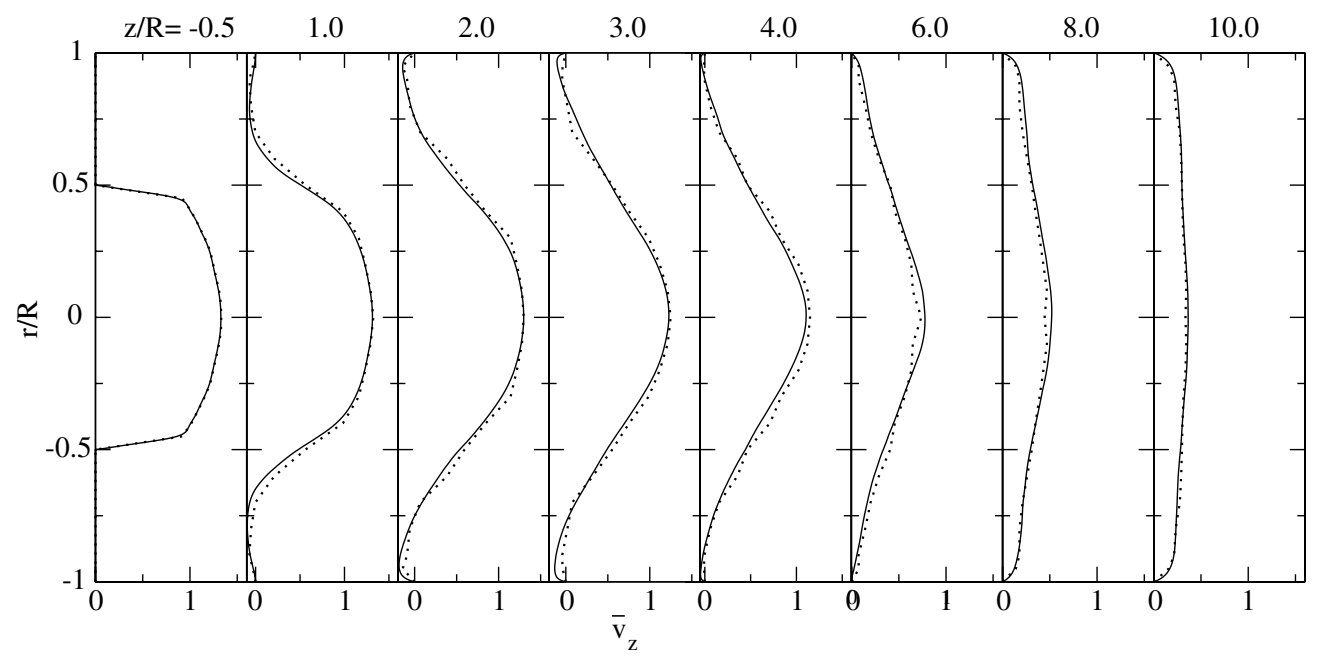

Fig. 2 Axial mean-velocity profile at different distances from the inlet of the main domain from the inlet of the main domain: experimental data (dotted lines) and LES data (solid lines); velocities (here and subsequently) are normalized against $V_{b}$.

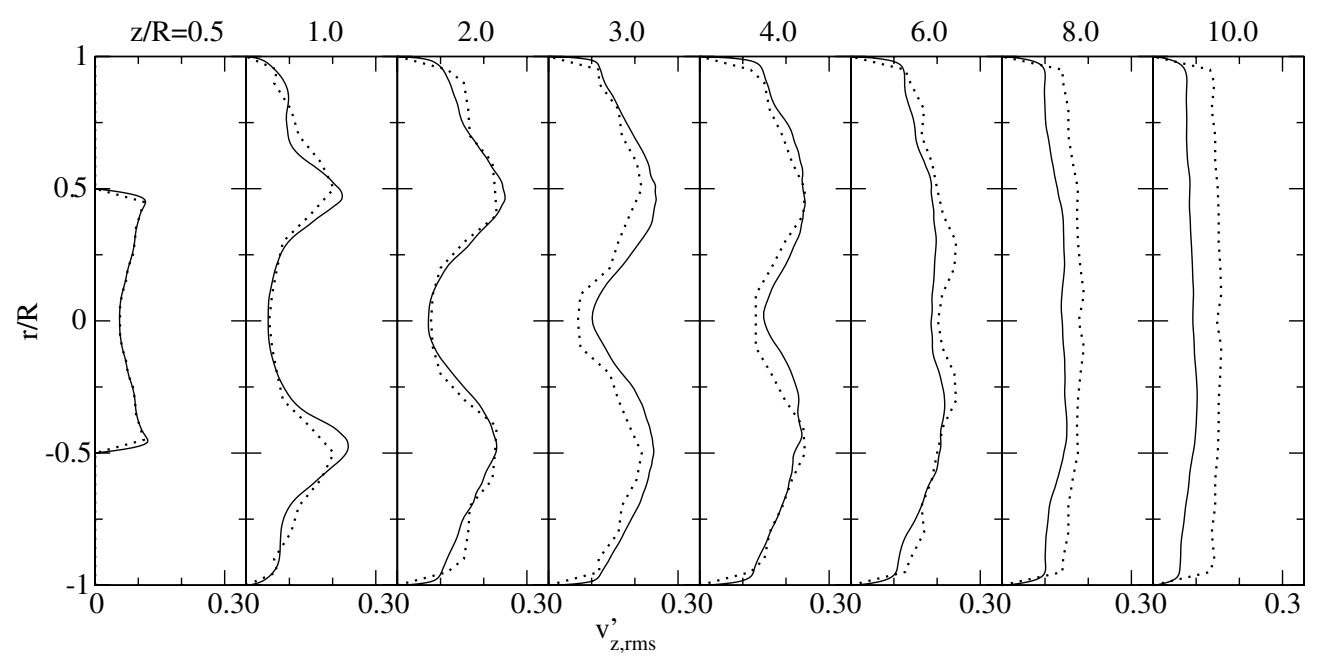

Fig. 3 Axial fluctuating rms velocity $v_{z, \text { rms }}$ at different distances from the inlet of the main domain from the inlet of the main domain: experimental data (dotted lines) and LES data (solid lines). 


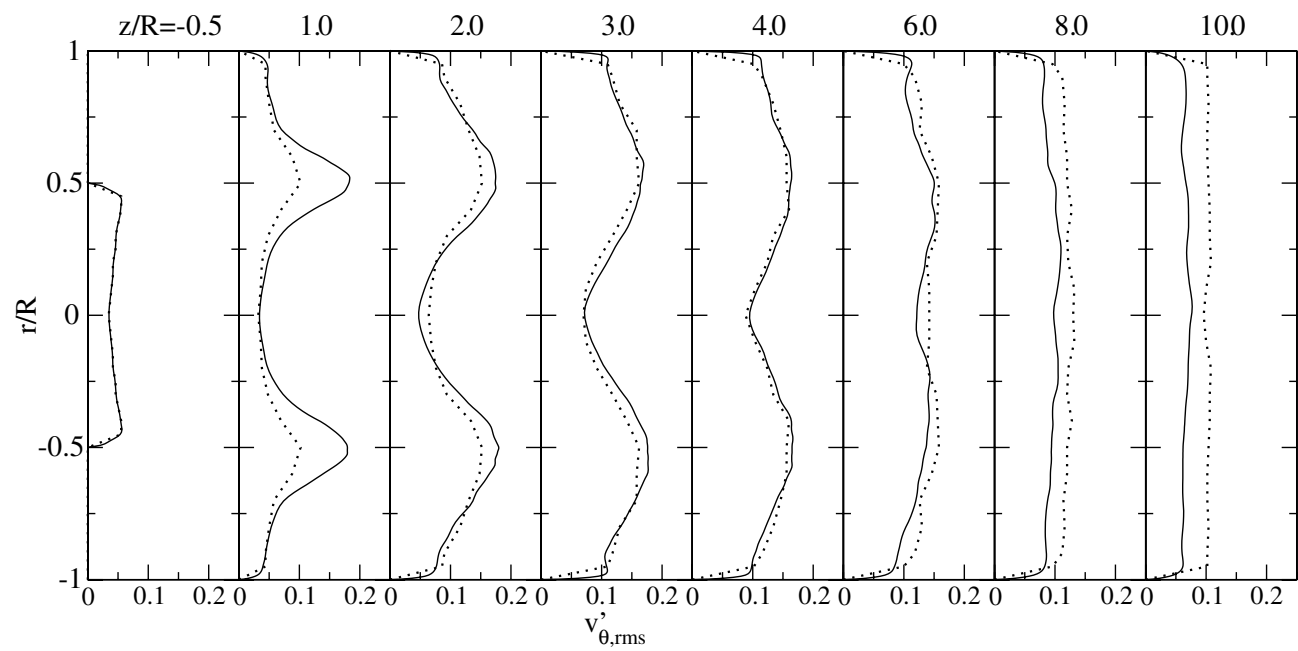

Fig. 4 Tangential fluctuating rms velocity $v_{\theta, \text { rms }}$ at different distances from the inlet of the main domain from the inlet of the main domain: experimental data (dotted lines) and LES data (solid lines).
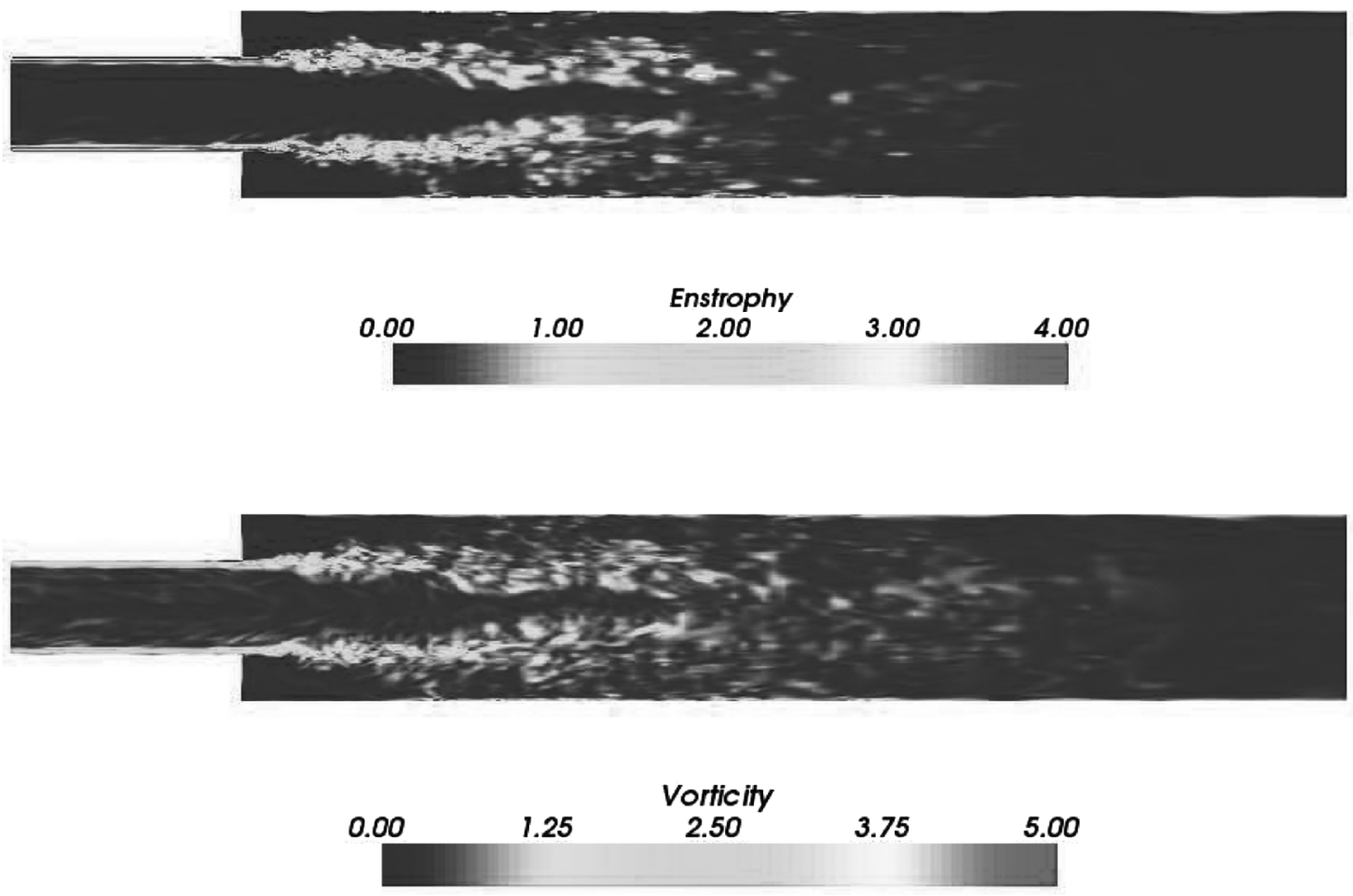

Fig. 5 Center-plane system plots of a) enstrophy and b) vorticity.

Pitsch [27] and Schlüter et al. [28]. For our calculation, a simple zerogradient outflow condition was used.

Contour plots for instantaneous snapshots of enstrophy and vorticity are also presented. Figures $5 \mathrm{a}$ and $5 \mathrm{~b}$ show that there is a smooth transfer of results from the mapping surface. The location of the mapping surface is shown in Fig. 1. There is no significant difference in the results across the mapping surface, and this verifies that the changes introduced in the equations in the mapping section to regenerate target results have no significant negative effects on the solution. Temporal energy spectra have also been generated based on velocity data sampled at specific locations in the mesh for every time step through the simulation (see Fig. 1 for sampling locations). These spectra are reproduced in Fig. 6 . The results show significant power in the low frequencies, corresponding to the large eddies and the resolved upper part of the power-law region of the turbulent cascade. The expected shape of the spectrum will be affected by the expansion, and at $z / R=0.0$, the form of the spectrum does seem to change, with enhanced lower frequencies that probably correspond to features of the recirculation behind the expansion; however, without measured experimental data to compare with, it is impossible to say how realistic the spectra are. Of importance is to ascertain if the inlet method is having any discernible negative impact on the spectra. The recycling of flow through the inlet section should produce a characteristic frequency of $0.075 \mathrm{~Hz}$. Examining the energy spectra does reveal a minor peak at this frequency up to $z / R=2.5$; however, it is a very small feature of the spectrum, comparable in size with other features in the plot, and is well outside the power-law range of the spectrum.

\section{B. Swirling Case}

Figures $7-10$ show the results for this computation. The results (solid lines) are compared with experimental results (dotted lines) and Schlüter et al. [10] results (dotted-dashed lines). In this case, despite some small discrepancies (especially near the edge of the expansion), the results agree very well with experimental data near 


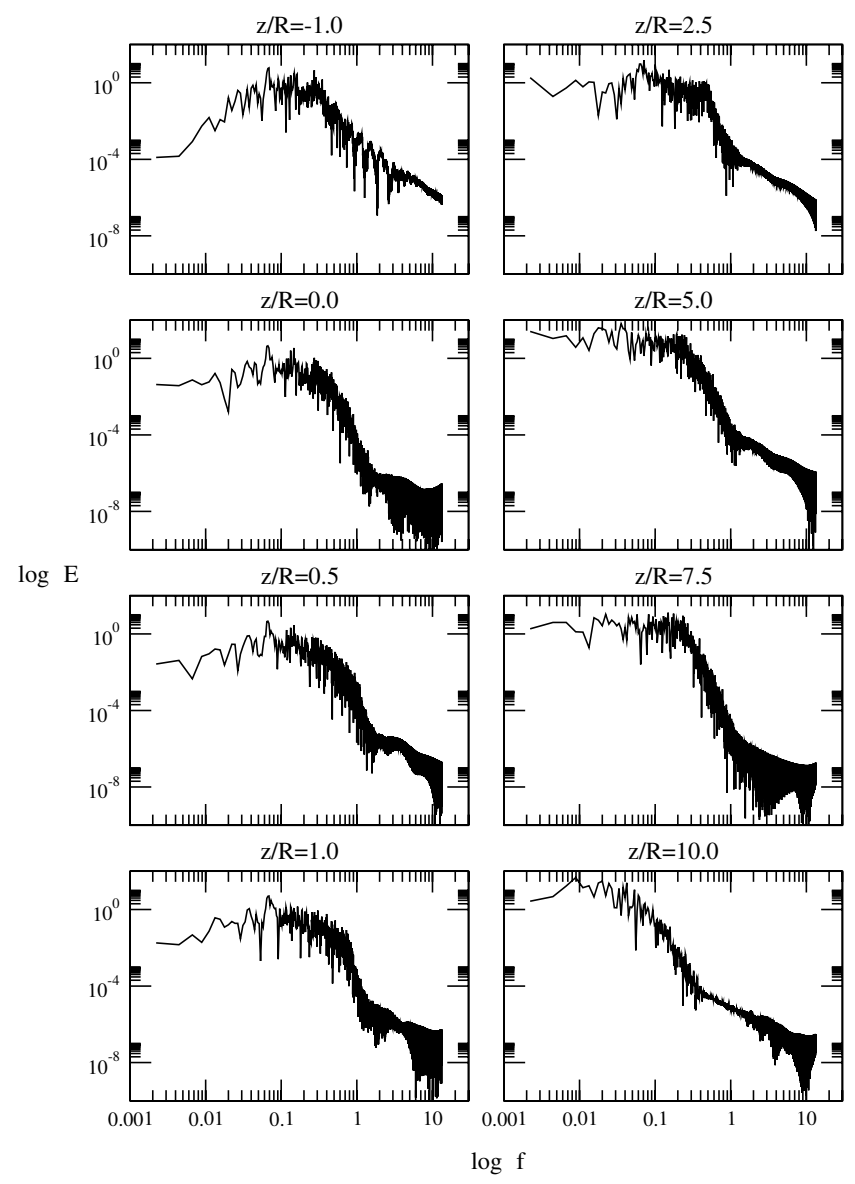

Fig. 6 Energy spectra generated from time-series data for parallel jet flow $(S=0.0)$ at different distances from the inlet of the main domain; frequency is measured in hertz.

the outlet, in contrast to the previous case $(S=0.0)$. This can be explained by the fact that the level of internal turbulence production due to the breakdown of the swirl in this type of flow is rather high behind the expansion; thus, the relative effect of turbulence entering the domain at the inlet is much less. The origin of the inner recirculation zone in highly swirling flows is fixed at the location of the expansion, which means that the zones of turbulence production (the shear layers created by the recirculating fluid and the issuing jet) are well-determined and independent of the inflow conditions. The turbulence level is then almost entirely defined by the turbulence production behind the step, which makes the flow almost independent of the inlet turbulence intensity. This case shows that situations exist in which the inlet turbulence plays a minor role, even when complex flow configurations are considered. In this special case, the high level of turbulence production inside the LES domain is dominant and its location and level are not determined by the inlet turbulence conditions. However, generating the correct mean (swirling) flow is still crucial in producing the correct results, and as can be seen from Figs. $\underline{7}$ and $\underline{8}$, our method is performing rather well for this.

Energy spectra have also been generated for this case (Fig. 11). In this case, the run was somewhat shorter, and so the spectrum shows less of the lowest frequencies; the frequency corresponding to the mapping frequency would only just be visible. The results are similar to those for the zero-swirl case, except that there is a distinct dropoff in power at the high end of the spectrum, corresponding to frequencies close to the cutoff, which is, of course, determined by the time step. Such an effect has been noticed elsewhere and has been attributed to a consequence of the implicit filtering used in finite volume LES [29]. That this effect did not appear for the zero-swirl case may indicate that our velocity-correction equation (4) may be generating slightly too much small-scale turbulence at the inlet; for the zero-swirl case, the turbulence in the domain is heavily affected by the inlet conditions and this effect may carry over. Running our method without the velocity correction generates results that demonstrate this dropoff. For the swirl case, the turbulence in the domain is principally generated by breakdown of the bulk swirl and so would not be as affected by the inlet flow.

\section{Analysis}

The mean flow in the body of the domain is generally wellreproduced by our LES. Some discrepancies are seen directly downstream of the expansion for the axial velocity for the swirl case (Fig. 7), but our method performs at least as well as the comparison LES data from Schlüter et al. [10]. The rms velocity profiles are also well-predicted, except for a short distance downstream of the expansion in the case of the no-swirl test case. In general, for all properties, our results are comparable with, or better than, the comparison LES data. In particular, no additional length of inlet is

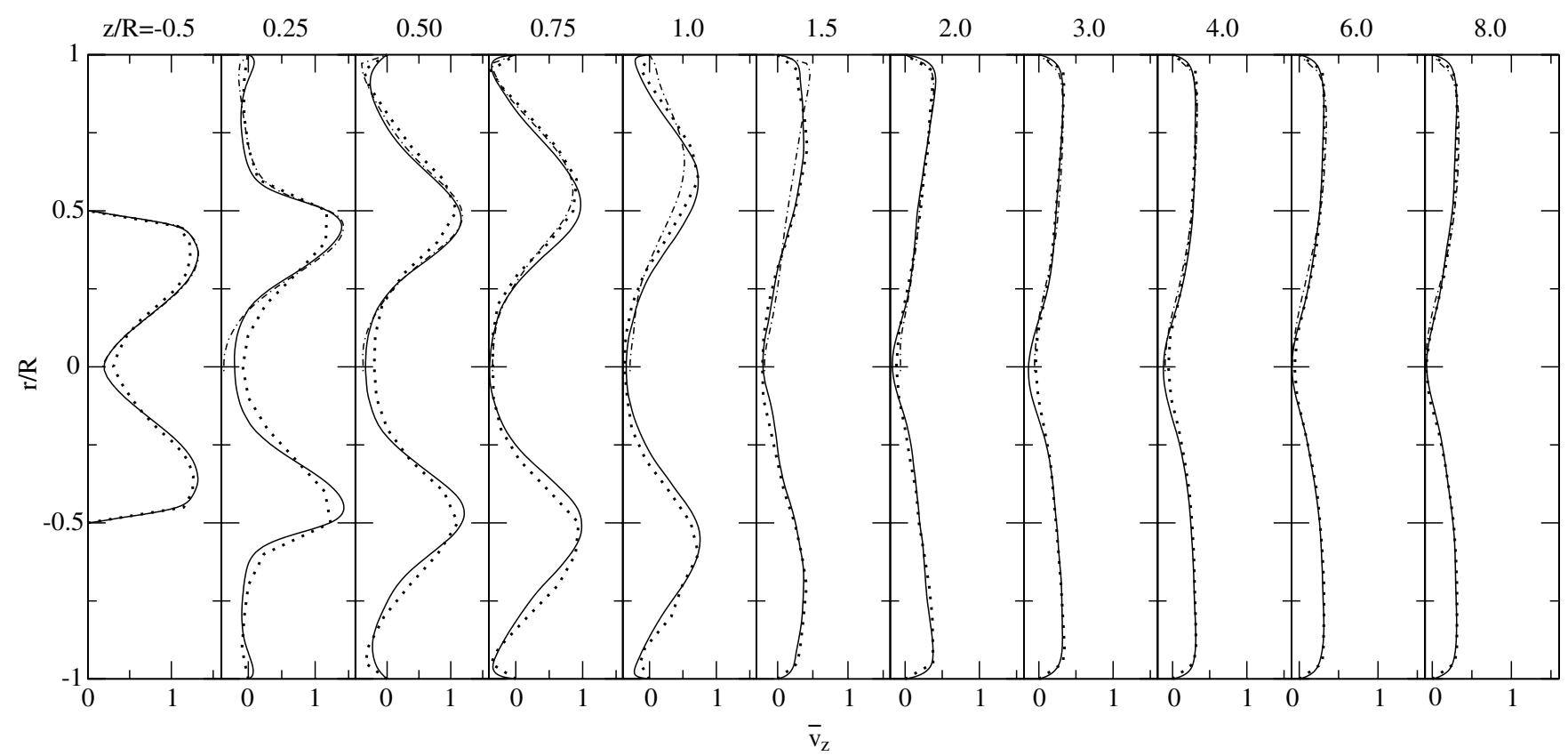

Fig. 7 Axial mean-velocity profile at different distances from the inlet of the main domain: experimental data (dotted lines), LES data (solid lines), and Schlüter et al. [10] data (dotted-dashed lines). 


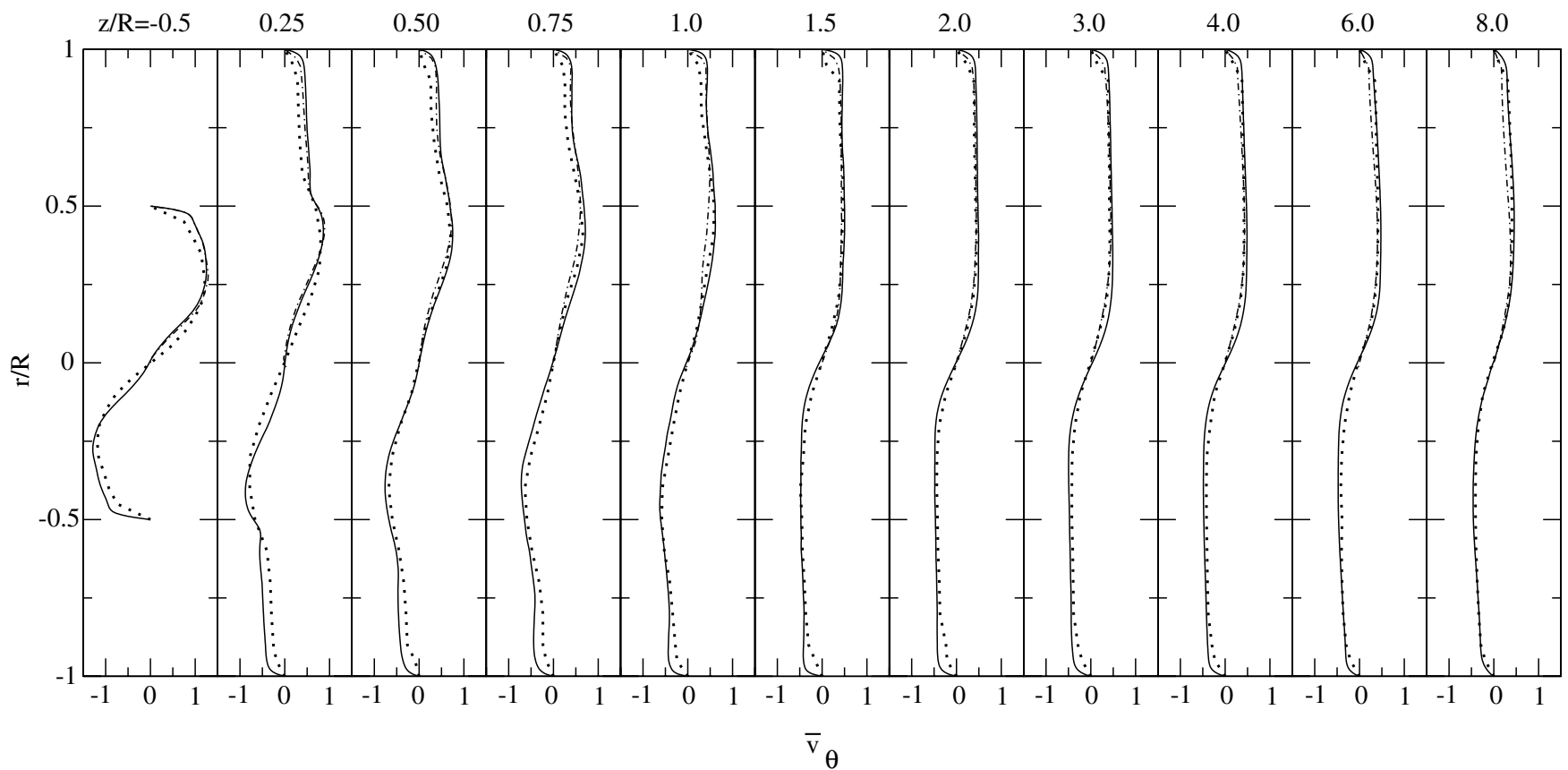

Fig. 8 Tangential mean-velocity profile at different distances from the inlet of the main domain: experimental data (dotted lines), LES data (solid lines), and Schlüter et al. [10] data (dotted-dashed lines).

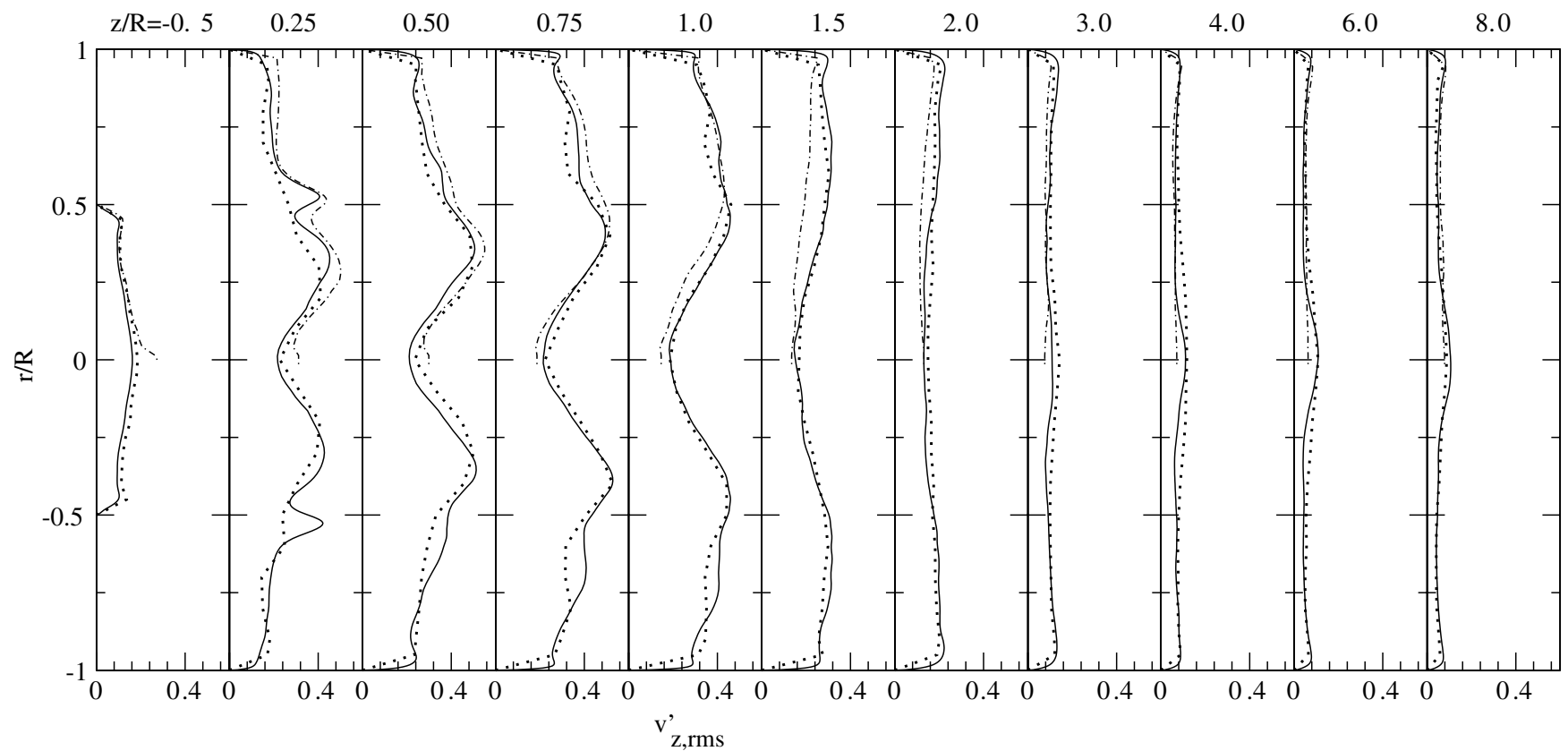

Fig. 9 Profiles of $v_{z, \text { rms }}^{\prime}$ at different distances from the inlet of the main domain: experimental data (dotted lines), LES data (solid lines), and Schlüter et al. [10] data (dotted-dashed lines).

required for the turbulent fluctuations to develop in any way, and there is no significant impact on the flow behavior in the inlet section due to the additional modeling in this region. The two test cases demonstrate significantly different behavior with respect to the inlet perturbations. As observed elsewhere [14], zero- and low-swirl cases are strongly sensitive to fluctuations in the inlet velocity, and inlet methods that do not account for this behavior (e.g., laminar or whitenoise inlets) fail to perform well at all. High-swirl cases are more dominated by the breakdown of the swirl vortex to turbulence and so are less sensitive to turbulence at the inlet; in fact, laminar and whitenoise inlet conditions can be shown to produce reasonable results, at least for the mean flow profiles [14]. However, it is still necessary to generate the mean swirl flow, which our method does efficiently and completely. A low-swirl case (e.g., $S=0.3$ ) would show our method in the best light, because this would require swirl inlet but still be sensitive to inlet turbulence; however, much less comparison data are available for this case. The behavior of our method at the two extremes (zero and large swirl) is still convincing. Thus, we conclude that our method is a significant improvement over laminar or whitenoise synthesis inlets for this case.

Compared with library lookup, our method performs well, but the differences are more nuanced. Our results for the swirl case are at least as good as those of Schlüter et al. [14] and are better in places. Part of this may be because we are able to base the target flow directly on the experimentally determined profiles, although if this data were not available there would be no reason why we could not develop $\mathbf{v}_{\text {des }}$ analytically for a desired swirl number. Additionally, our method is simpler to apply. For a library-lookup method, a suitable auxiliary 


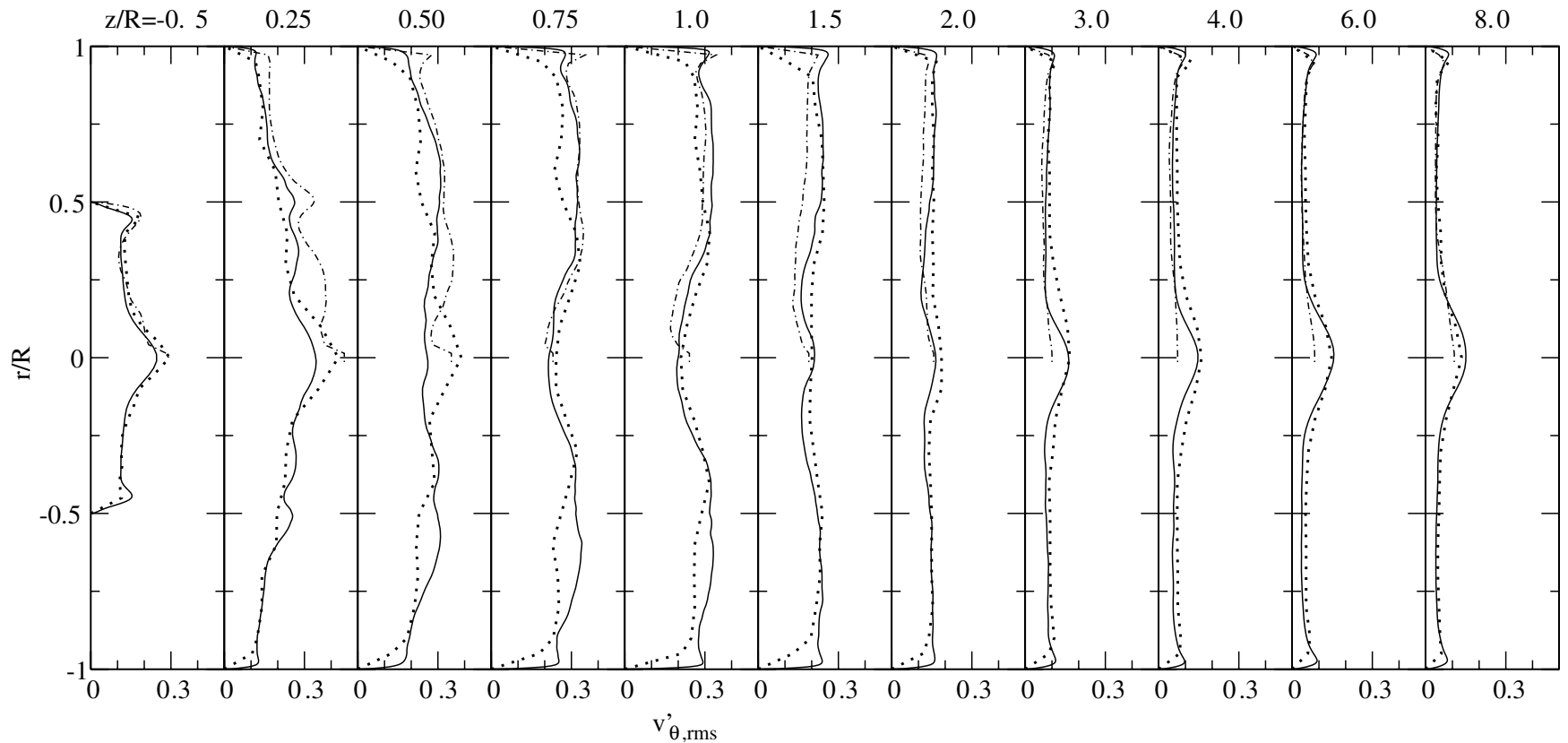

Fig. 10 Profiles of $v_{\theta, \mathrm{rms}}^{\prime}$ at different distances from the inlet of the main domain: experimental data (dotted lines), LES data (solid lines), and Schlïter et al. [10] data (dotted-dashed lines).

calculation has to be set up and simulated; the data must be stored for use, and recycling this data can introduce unwanted periodic behavior into the flow and restrict the time span used for generating flow statistics in the main domain. Running the auxiliary

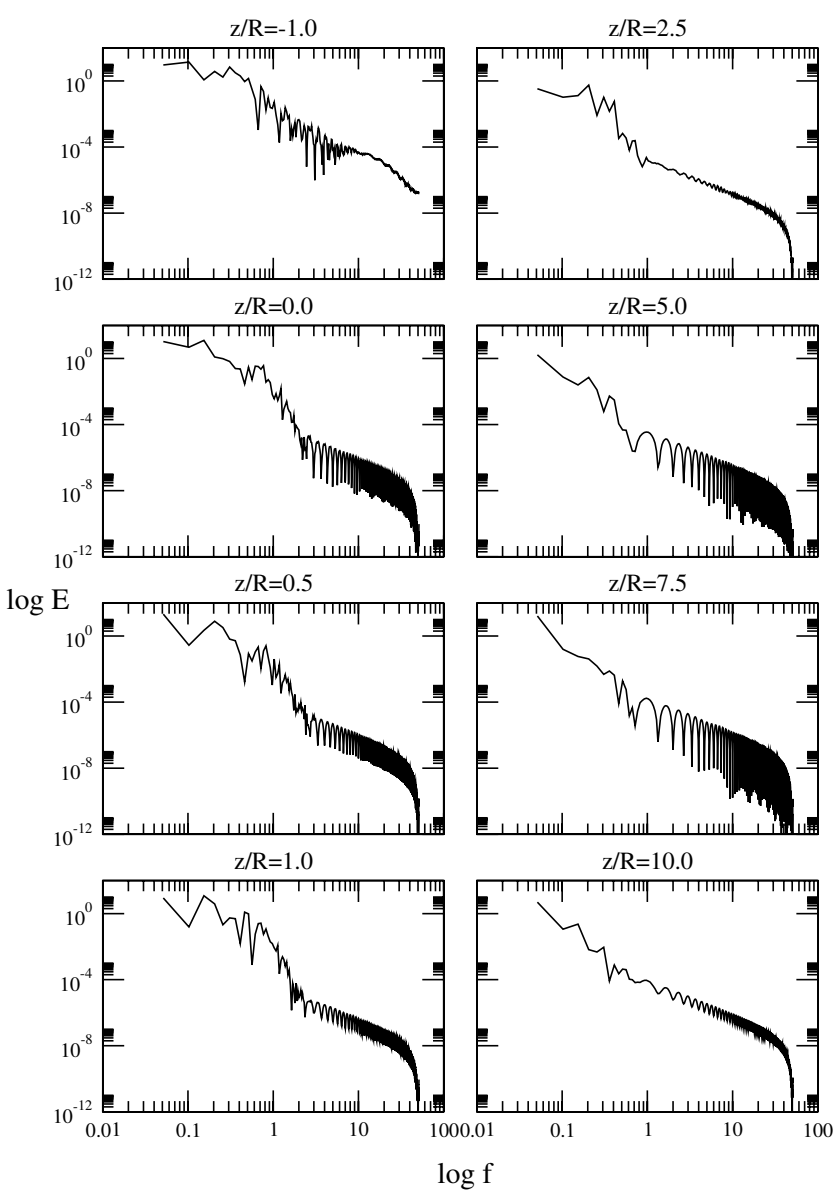

Fig. 11 Energy spectra generated from time-series data for a jet with strong swirl $(S=0.6)$ at different distances from the inlet of the main domain. computation at the same time as the main calculation obviates these problem, but introduces its own programming problems. Additionally, if the flow conditions change (e.g., a different Reynolds number), then the library must be recomputed or rescaled; the latter can be shown to introduce additional problems into the simulation [5]. Integrating the auxiliary computation into the main domain in this manner, on the other hand, is much more elegant and easier to manage and generates extremely good mean and turbulent flow results. Although the recycling of flow through the inlet section will introduce some degree of periodicity, successive passes through the inlet section are not identical and so the periodic effects are much less than would be the case for a library method. The relative computational costs of the different methods may vary according to the exact problem being simulated. If the main domain needs to be extended to provide an inlet section for our mapping method, then this will impose an increased computational requirement on the main calculation, which would be comparable with the cost of running an auxiliary calculation. Indeed, if the auxiliary calculation can be reduced in scale (e.g., by storing a short data set or using a longer time step), then this might reduce the computational cost for the library method. However, these steps impose problems of their own (for instance, interpolation between saved time steps if a longer time step is used for the auxiliary calculation). For our mapping case presented here, the inlet cells represent approximately $5 \%$ of the total, and so the computational costs associated with these additional cells are relatively minor. Additionally, the inclusion of the additional and corrective terms into the Navier-Stokes equations for the inlet section does seem to have little negative impact on the flow in this region, and so it may be possible for the mapping to take place on a section of mesh that is part of the desired physical domain, not simply an additional extension to the computational domain.

\section{Conclusions}

In this paper, we present a novel technique for generating swirl inlets for LES. The technique involves introducing an artificial body force and velocity-correction terms into the early part of the computational domain (designated as the inlet section) with remapping of the velocity from the end of the inlet section back to the domain inlet. Control algorithms are used to vary the body force and velocity correction to generate desired swirl, mean-velocity, and Reynolds-stress profiles. The method was applied to flow in an axisymmetric sudden expansion, with and without swirl at the inlet, 
and was compared against experimental and literature LES data and against similar results in the literature. The method generates excellent results for this case, in particular, matching mean and fluctuating components of the velocity very precisely for both swirling and nonswirling cases. We find no negative impact on the turbulence properties as evidenced from the enstrophy or the temporal power spectra. Finally, the method is elegant and straightforward to implement.

\section{Acknowledgments}

G. R. Tabor and M. H. Baba-Ahmadi acknowledge the support of the Engineering and Physical Sciences Research Council through grant GR/R27495/01. We would like to thank N. Swaminathan for computer time to perform the computations and J. U. Schlüter and P. A. Dellenback for access to their large eddy simulation and experimental results, respectively. We would also like to thank the referees for their valuable comments.

\section{References}

[1] Jakirlić, S., Hanjalić, K., and Tropea, C., "Modelling Rotating and Swirling Turbulent Flows: A Perpetual Challenge," AIAA Journal, Vol. 40, No. 10, 2002, pp. 1984-1996.

[2] Pope, S. B., "Ten Questions Concerning the Large-Eddy Simulation of Turbulent Flows," New Journal of Physics, Vol. 6, No. 35, 2004, doi: 10.1088/1367-2630/6/1/035.

[3] Huang, Y., and Yang, V., "Effect of Swirl on Combustion Dynamics in a Lean-Premixed Swirl-Stabilized Combustor," Proceedings of the Combustion Institute, Vol. 30, Combustion Inst., Pittsburgh, PA, 2005, pp. $1775-1782$. doi:10.1016/j.proci.2004.08.237

[4] Wang, S., Yang, V., Hsiao, G., Hsieh, S.-Y., and Mongia, H. C., "Large-Eddy Simulations of Gas-Turbine Swirl Injector Flow Dynamics," Journal of Fluid Mechanics, Vol. 583, 2007, pp. 99-122. doi:10.1017/S0022112007006155

[5] Wang, P., and Bai, X., "Large Eddy Simulations of Turbulent Swirling Flows in a Dump Combustor: A Sensitivity Study," International Journal for Numerical Methods in Fluids, Vol. 47, No. 2, 2005, pp. 99 120.

doi:10.1002/fld.781

[6] Lee, S., Lele, S., and Moin, P., "Simulation of Spatially Evolving Turbulence and the Applicability of Taylor's Hypothesis in Compressible Flow," Physics of Fluids A, Vol. 4, No. 7, 1992, pp. 1521-1530. doi: $10.1063 / 1.858425$

[7] Andersson, N., Eriksson, L.-E., and Davidson, L., "Effects of Inflow Conditions and Subgrid Model on LES for Turbulent Jets," 11th AIAA/ CES Aeroacoustics Conference, Monterey, CA, AIAA Paper 20052925, May 2005.

[8] Di Mare, L., Klein, M., Jones, W. P., and Janicka, J., "Synthetic Turbulence Inflow Conditions for Large Eddy Simulation," Physics of Fluids, Vol. 18, No. 2, 2006, pp. 1-11. doi:10.1063/1.2130744

[9] Lund, T. S., Wu, X., and Squires, K. D., "Generation of Turbulent Inflow Data for Spatially Developing Boundary Layer Simulations," Journal of Computational Physics, Vol. 140, No. 2, Mar. 1998, pp. 233-258. doi:10.1006/jeph.1998.5882

[10] Schlüter, J. U., Pitsch, H., and Moin, P., "Large Eddy Simulation Inflow Conditions for Coupling with Reynolds-Averaged Flow Solvers," AIAA Journal, Vol. 42, No. 3, Mar. 2004, pp. 478-484. doi: $10.2514 / 1.3488$

[11] Pierce, C. D., and Moin, P., "Large Eddy Simulation of a Confined Coaxial Jet with Swirl and Heat Release," AIAA Paper 98-2892, Juen 1998

[12] Wang, P., Bai, X. S., Wessman, M., and Klingmann, J., "Large Eddy Simulation and Experimental Studies of a Confined Turbulent Swirling Flow," Physics of Fluids, Vol. 16, No. 9, 2004, pp. 3306-3324. doi:10.1063/1.1769420

[13] Garcia-Villalba, M., and Fröhlich, J., "LES of a Free Annular Swirling Jet Dependence of Coherent Structures on a Pilot Jet and the Level of Swirl," International Journal of Heat and Fluid Flow, Vol. 27, No. 5, 2006, pp. 911-923.

doi:10.1016/j.ijheatfluidflow.2006.03.015

[14] Schlüter, J. U., Pitsch, H., and Moin, P., "Boundary Conditions for LES in Coupled Simulations," 41st AIAA Aerospace Sciences Meeting, AIAA Paper 2003-0069, Jan. 2003.

[15] Tabor, G., Baba-Ahmadi, M. H., de Villiers, E., and Weller, H. G., "Construction of Inlet Conditions for LES of Turbulent Channel Flow," Proceedings of the ECCOMAS Congress [CD-ROM], Univ. of Jyväskylä, Jyväskylä, Finland 2004.

[16] De Villiers, E., "The Potential For Large Eddy Simulation for the Modelling of Wall Bounded Flows," Ph.D. Thesis, Imperial College, London, 2006

[17] Fureby, C., Tabor, G., Weller, H., and Gosman, A. D., "A Comparative Study of Sub Grid Scale Models in Homogeneous Isotropic Turbulence," Physics of Fluids, Vol. 9, No. 5, 1997, pp. 1416-1429. doi:10.1063/1.869254

[18] Ghosal, S., Lund, T. S., Moin, P., and Akselvoll, K., "A Dynamic Localization Model for Large Eddy Simulation of Turbulent Flows," Journal of Fluid Mechanics, Vol. 286, Mar. 1995, pp. 229-255. doi:10.1017/S0022112095000711

[19] Fureby, C., Gosman, A. D., Tabor, G., Weller, H. G., Sandham, N., and Wolfshtein, M., "Large Eddy Simulation of Turbulent Channel Flows," Turbulent Shear Flows 11, Vol. 3, Springer-Verlag, New York, 1997, pp. 19-30.

[20] Fureby, C., Tabor, G., Weller, H. G., and Gosman, A. D., "Differential Subgrid Stress Models in Large Eddy Simulations," Physics of Fluids, Vol. 9, No. 11, 1997, pp. 3578-3580. doi: $10.1063 / 1.869467$

[21] Fureby, C., Tabor, G., Weller, H. G., and Gosman, A. D., "Large Eddy Simulation of the Flow Around a Square Prism," AIAA Journal, Vol. 38, No. 3, 2000, pp. 442-452.

[22] Jasak, H., Weller, H., and Gosman, A., "High Resolution NVD Differencing Scheme for Arbitrarily Unstructured Meshes," International Journal for Numerical Methods in Fluids, Vol. 31, No. 2, 1999, pp. 431-449.

doi:10.1002/(SICI) 1097-0363(19990930)31:2<431::AIDFLD884>3.0.CO;2-T

[23] Rhie, C. M., and Chow, W. L., "Numerical Study of the Turbulent Flow Past an Airfoil with Trailing Edge Separation," AIAA Journal, Vol. 21, No. 11, 1983, pp. 1525-1532.

[24] Issa, R. I., "Solution of the Implicitly Discretised Fluid Flow Equations by Operator-Splitting," Journal of Computational Physics, Vol. 62, No. 1, 1986, pp. 40-65. doi:10.1016/0021-9991(86)90099-9

[25] Dellenback, P. A., Metzger, D. E., and Neitzel, P., "Measurements in Turbulent Swirling Flow Through an Abrupt Axisymmetric Expansion," AIAA Journal, Vol. 26, No. 6, 1988, pp. 669-681.

[26] Moin, P., "Progress in Large Eddy Simulation of Turbulent Flows," AIAA Paper 97-0749, 1997.

[27] Schlüter, J. U., and Pitsch, H., "Consistent Boundary Conditions for Integrated LES/RANS Simulations: LES Outflow Conditions," Annual Research Briefs 19-30, NASA Ames/Stanford Univ., Center for Turbulence Research, Stanford, CA, 2001.

[28] Schlüter, J. U., Pitsch, H., and Moin, P., "Outflow Conditions for Integrated Large Eddy Simulation/Reynolds-Averaged Navier-Stokes Simulations," AIAA Journal, Vol. 43, No. 1, Jan. 2005, pp. 156-164. doi:10.2514/1.11007

[29] De Stefano, G., and Vasilyev, O. V., "A Study of the Effect of Smooth Filtering in LES," Proceedings of the Third AFOSR International Conference on Direct Numerical Simulation and Large Eddy Simulation (TAICDL), Kluwer Academic, Norwell, MA, 2001, Paper 18. 